\title{
Evaluation of a new approach for semi-automatic segmentation of the cerebellum in patients with multiple sclerosis
}

\author{
Katrin Weier • Andreas Beck $\cdot$ Stefano Magon • Michael Amann • Yvonne Naegelin • \\ Iris K. Penner $\cdot$ Markus Thürling $\cdot$ Volker Aurich $\cdot$ Tobias Derfuss $\cdot$ Ernst-Wilhelm Radue \\ Christoph Stippich $\cdot$ Ludwig Kappos $\cdot$ Dagmar Timmann $\cdot$ Till Sprenger
}

Received: 22 March 2012/Revised: 17 May 2012/ Accepted: 19 May 2012/Published online: 19 June 2012

(c) Springer-Verlag 2012

\begin{abstract}
Cerebellar dysfunction is an important contributor to disability in patients with multiple sclerosis (MS), however, few in vivo studies focused on cerebellar volume loss so far. This relates to technical challenges regarding the segmentation of the cerebellum. In this study, we evaluated the semi-automatic ECCET software for performing cerebellar volumetry using high-resolution 3D
\end{abstract}

K. Weier $(\bowtie) \cdot$ S. Magon · M. Amann · Y. Naegelin .

T. Derfuss · L. Kappos · T. Sprenger

Department of Neurology, University Hospital Basel,

Petersgraben 4, 4031 Basel, Switzerland

e-mail: weierk@uhbs.ch

A. Beck

Beck Datentechnik, 40764 Langenfeld, Germany

M. Amann · C. Stippich · T. Sprenger

Division of Neuroradiology, Department of Radiology

and Nuclear Medicine, University Hospital Basel,

4031 Basel, Switzerland

I. K. Penner

Department of Cognitive Psychology and Methodology,

University of Basel, 4055 Basel, Switzerland

M. Thürling · D. Timmann

Department of Neurology, University of Duisburg-Essen,

45122 Essen, Germany

M. Thürling

Erwin L. Hahn Institute for Magnetic Resonance Imaging,

University of Duisburg-Essen, Essen, Germany

V. Aurich

Department of Computer Sciences, University of Düsseldorf, Düsseldorf, Germany

E.-W. Radue

Medical Image Analysis Center, University Hospital Basel, 4031 Basel, Switzerland
T1-MR scans in patients with MS and healthy volunteers. We performed test-retest as well as inter-observer reliability testing of cerebellar segmentation and compared the ECCET results with a fully automatic cerebellar segmentation using the FreeSurfer software pipeline in $15 \mathrm{MS}$ patients. In a pilot matched-pair analysis with another data set from 15 relapsing-remitting MS patients and 15 ageand sex-matched healthy controls (HC), we assessed the feasibility of the ECCET approach to detect MS-related cerebellar volume differences. For total normalized cerebellar volume as well as grey and white matter volumes, intrarater (intraclass correlation coefficient $($ ICC) $=0.99$, $95 \% \mathrm{CI}=0.98-0.99)$ and interobserver agreement (ICC $=0.98,95 \% \mathrm{CI}=0.74-0.99)$ were strong. Comparison between ECCET and FreeSurfer results likewise yielded a good intraclass correlation (ICC $=0.86,95 \%$ $\mathrm{CI}=0.58-0.95)$. Compared to HC, MS patients had significantly reduced normalized total brain, total cerebellar, and grey matter volumes $(p \leq 0.05)$. ECCET is a suitable tool for cerebellar segmentation showing excellent testretest and inter-observer reliability. Our matched-pair analysis between MS patients and healthy volunteers suggests that the method is sensitive and reliable in detecting cerebellar atrophy in MS.

Keywords Multiple sclerosis - Cerebellar atrophy . Volumetry · Grey matter

\section{Introduction}

The cerebellum plays an important role in motor coordination and motor learning. In addition, the cerebellum most likely supports cognitive processes, especially attention. It receives input from afferent sensory systems as well as from 
other parts of the brain and integrates these inputs to fine tune motor activity and possibly non-motor function such as cognition, emotion, and behavior [1,2]. In multiple sclerosis (MS), cerebellar dysfunction is a significant contributor to the development of disability and often progresses despite disease-modifying treatment [3, 4]. In spite of the wellknown importance of the cerebellum in MS, only limited data on cerebellar white (WM) or grey matter (GM) volume loss are available. MRI studies have confirmed extensive GM demyelination in the cerebellum $[5,6]$ especially in patients with a secondary-progressive disease course compared to patients with benign MS [7]. These studies focused on cross-sectional data and did not investigate the changes of cerebellar grey and white matter volume (WMV) over time. The fact that so few studies have investigated cerebellar volume using MRI relates to technical challenges regarding the correct segmentation and extraction of the cerebellar tissue from nearby structures such as the peduncles, brainstem, spinal cord, and venous sinuses. Furthermore, its thin gyri and sulci are difficult to segment due to partial volume effects and B1-field inhomogeneities, which impair the tissue contrast. In addition, 2D MR acquisitions with limited volume coverage might hinder full cerebellar imaging depending of the size of the brain.

Different strategies for cerebellar segmentation and volume measurements have been proposed in the past, but so far, no gold standard has been established. FreeSurfer (http://surfer.nmr.mgh.harvard.edu) [8] is an automatic approach, which has been frequently used in recent years for cortical segmentation and cortical thickness analysis, but especially in smaller structures such as the hippocampus, FreeSurfer has been shown to be limited [9]. SánchezBenavides et al. [10] for example pointed out that results from automated hippocampal volumetry using FreeSurfer showed a tendency to overestimate the volume when compared to manual tracing. The semiautomatic software "ECCET" has proven to be useful when segmenting the cerebellum in degenerative cerebellar disorders [e.g., spinocerebellar ataxia type 6 (SCA6), sporadic adult-onset ataxia (SAOA)] [11, 12], but so far the ECCET approach has not been systematically evaluated in MS patients.

The aim of this study was to test the reliability of ECCET, to compare ECCET against FreeSurfer, and to study the feasibility of the method for detecting cross-sectional group differences between MS patients and healthy controls.

\section{Materials and methods}

Reliability study and comparison with FreeSurfer

Test-retest and inter-observer reliability testing of ECCET and a comparison to the fully automatic segmentation program FreeSurfer were performed using high-resolution T1-weighted MPRAGE images acquired in sagittal plane $\left(\mathrm{TR} / \mathrm{TI} / \mathrm{TE}=2,080 / 1,100 / 3.0 \mathrm{~ms} ; \quad \alpha=15^{\circ}, 160\right.$ slices, isotropic voxel of $1 \mathrm{~mm}^{3}$ ). In addition, axial 3-mm proton density-weighted (PDw) and T2-weighted (T2w) images were acquired to determine the cerebral and cerebellar lesion load in MS patients (double spin echo: TR/TE1/ TE2 $=3,980 / 14 / 108 \mathrm{~ms} ; 40$ slices with an in-plane resolution of $1 \times 1 \mathrm{~mm}$ ). These MRI scans were performed at the Department of Radiology, Division of Neuroradiology, University Hospital Basel, Switzerland using a $1.5 \mathrm{~T}$ Magnetom Avanto (Siemens Medical Solutions, Erlangen, Germany). Data from 15 randomly selected MS patients (six women, mean age 49 years, range 31-67 years) taking part in an ongoing cohort study on the genotypic-phenotypic characterization of MS were included. All patients underwent a medical and neurological examination, including a structured assessment of the Expanded Disability Status Scale (EDSS) [13] and review of the patient's past medical history. The selected patients had a mean disease duration of 13 years (range 5-23 years) and a mean EDSS score of 2.9 (range 0-6). The mean cerebellar functional system score (FSS) was 1.4 (range 0-3). Twelve patients (five women) had a relapsing-remitting (RRMS) disease course, two patients (one woman) were secondary progressive (SPMS) and one man had been diagnosed with primary progressive MS (PPMS). Written informed consent was obtained before the examination and the study was approved by the local ethics committee.

\section{Matched-pair analysis}

The MRI data for the comparison between healthy controls (HC) and MS patients were acquired at a 3-T Magnetom Verio (Siemens Medical Solutions, Erlangen, Germany). A high-resolution T1-weighted MPRAGE sequence was used for the cerebellar segmentation $(\mathrm{TR} / \mathrm{TI} / \mathrm{TE}=1,570 / 900 /$ $2.7 \mathrm{~ms}, \alpha=9^{\circ}, 160$ sagittal slices, isotropic voxel of $1 \mathrm{~mm}^{3}$ ). Axial 3-mm proton PDw and T2w scans were additionally used for determining the PD/T2 lesion load (double spin echo: TR/TE1/TE2 $=3,500 / 9.1 / 91 \mathrm{~ms} ; 40$ slices with an in-plane resolution of $0.75 \times 0.75 \mathrm{~mm}$ ). All MRI scans were acquired at the Department of Radiology, Division of Neuroradiology, University Hospital Basel, Switzerland. All patients underwent a thorough medical and neurological examination, including the structured assessment of the EDSS and review of the patients' past medical histories. Fifteen patients (ten women, mean age 35 years, range 22-62 years) with a relapsing-remitting disease course were studied, who had an overall disease duration of 8 years (range 1-17 years) and a mean EDSS score of 2.7 (range 1-4.5). The mean cerebellar FSS was 0.9 (range $0-3$ ) in this patient group. The patients were 
compared to 15 age- and gender-matched HC (mean age 35 years, range $22-62$ years). Written informed consent was provided before the examination and the study was approved by the local ethics committee.

Data postprocessing and statistical analysis

An identical protocol was carried out for the volumetric analysis in all parts of the study.

The high-resolution T1-weighted MPRAGE datasets were first reoriented to the AC-PC plane (with reslicing using cubic spline interpolation, final voxel size $1 \times 1 \times 1 \mathrm{~mm})$. This was carried out using Brainvoyager QX (Brain Innovations, Maastricht, The Netherlands) [14]. After this initial step, all subsequent processing steps were performed using a structured and guided workflow with the ECCET toolkit (http://www.eccet.de), which had been adapted for this study.

An edge-preserving non-linear Gaussian noise reduction filter was applied to the isotropic 3D T1-MPRAGE MRI volumes $[15,16]$. In order to achieve a high reproducibility of cerebellar volumetry, an exact and reproducible separation of the brainstem from the cerebellar peduncles is necessary. This issue relates to the relatively poor definition of the borders between the brainstem and the cerebellar peduncles on conventional MRI. Therefore, we manually outlined the brainstem on five or more slices: on one slice superior as well as inferior to the cerebellar peduncles and on at least two slices at the pontine level according to anatomical landmarks, such as the trigeminal and vestibulo-cochlear nerve entry zones (Fig. 1). The manually traced regions of interest were then connected using interpolation based on an angle-restricted convex hull generation between the segmented slices. In this algorithm, the center of gravity of the segmentation for each slice is searched and the border points of each slice are then connected with their corresponding border points on the neighboring slices. Next, a semi-automatic 3D filling algorithm differentiating between brain tissue and surrounding cerebrospinal fluid (CSF) was applied $[17,18]$. We used a four-step gap-tolerant 3D filling algorithm with the following steps: (1) Building a distance field that, for any voxel of the volume, gives the distance to any part of the boundary object. (2) Filling the distance field from the inside (as defined by the user), stopping at a distance of five voxels from the boundary object. (3) Adding five extra layers using a dilation step that is matched to the metric used to build the distance field. (4) An additional pocketfilling step is performed, filling gaps, which remained unfilled in the previous steps. This is done by adding a series of 2D filling steps, which are reversed if the filled planes continue for more than ten steps, thus avoiding cases where the filling would spread into the outside non-brain space. This step is repeated for all three primary orientation planes and repeated three times.

Using these steps, a 3D volume of the cerebellum was generated, which was visually inspected and manually adjusted when required (Fig. 2). The total cerebellar volume (TCV) was then calculated on the basis of the number of cerebellar voxels. The segmentation between grey and white matter was based on a histogram analysis, where two major peaks of intensities (representing the grey and white matter peaks) are detected using a hat operator, which rejects very small maxima occurring due to image noise. The mean value between these peaks is then selected as the threshold distinguishing between white and grey matter. MRI visible MS lesions are mostly located in the white matter and often possess low image intensity on
Fig. 1 The manually outlined contour of the midbrain, pons, and medulla on five slices superior and inferior to the peduncles (blue) is used for the subsequent automatic interpolation (pink) of the brainstem

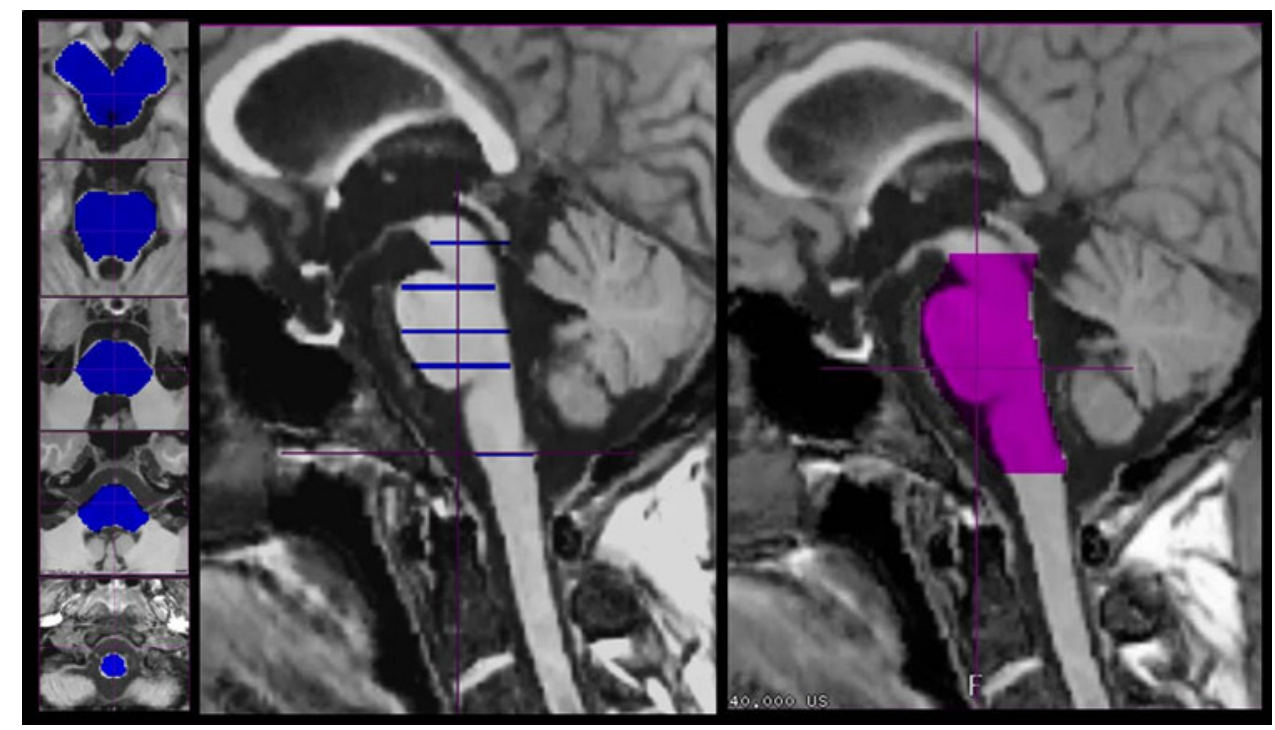




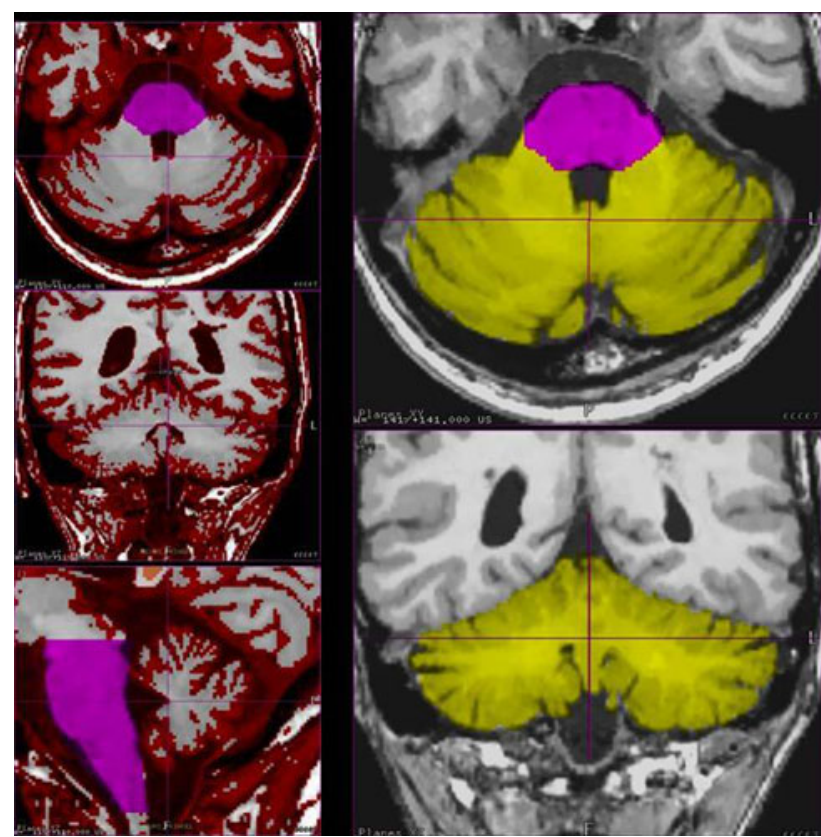

Fig. 2 Application of the 3D-filling algorithm (red) for the separation of brain tissue and surrounding cerebrospinal fluid (CSF). The 3D volume of the cerebellum (yellow) is visually inspected and manually adjusted when needed

T1-weighted images (so-called "black holes"). Such areas may potentially be misclassified as grey matter during the segmentation process. Lesion masking is therefore necessary for white versus grey matter segmentation. We used whole-brain lesion masks (generated on $\mathrm{PD} / \mathrm{T} 2$-weighted images as previously described [19]) and coregistered them to the 3D MPRAGE datasets using a mutual-information based registration-algorithm in ECCET in order to exclude corresponding regions of signal alteration during the cerebellar white matter/grey matter segmentation. T1w hypointense lesions usually possess a PD/T2w correlate enabling the use of $\mathrm{PD} / \mathrm{T} 2 \mathrm{w}$ lesion files for the masking of T1w datasets.

The normalized whole brain volume was calculated using SIENAX as previously described [20]. The SIENAX normalization factor (i.e., normalization for head size) was used for normalizing the cerebellar volumes, which were determined with ECCET.

For examination of test-retest reliability (TR-R) and interobserver reliability (IO-R), the protocol was carried out in each patient by two independent examiners twice each on two consecutive days. Both examiners underwent a training session segmenting ten different datasets beforehand. The mean values detailed below are averages of all measurements of both raters. Furthermore, ECCET was compared to the fully automated segmentation software FreeSurfer (http://surfer.nmr.mgh.harvard.edu) using the imaging data, which were also used for the reliability testing. The FreeSurfer procedure was applied as previously described [8]. Intraclass correlation coefficient (ICC) model 3 (mixed-effect model) for TR-R and ICC model 2 (random-effect model) for IO-R were calculated based on the agreement computation analysis [21,22] using SPSS 19 (IBM, New York, USA).

In the cross-sectional matched-pair analysis, one-sided $t$ tests were used for the group comparison between $\mathrm{HC}$ and MS patients. Additionally, multilinear regression was calculated between cerebellar volume and several clinical measures for the patient group. A $p$ value of $<0.05$ was used for statistical thresholding of significance.

\section{Results}

Reliability study and comparison with FreeSurfer

Analysis showed a mean TCV of $143 \mathrm{~cm}^{3}$ (standard deviation (SD) \pm 14 , range 117-174), a mean total cerebellar WMV of $22 \mathrm{~cm}^{3}(\mathrm{SD} \pm 5$, range 15.6-35) and a mean total cerebellar grey matter volume (GMV) of $121 \mathrm{~cm}^{3}$ (SD \pm 11 , range 104-139). For normalized cerebellar volumes, please see Table 1 . The mean normalized whole-brain volume (SIENAX analysis) was $1,536 \mathrm{~cm}^{3}$. The T2 lesion volumes ranged between 0.4 and $12.3 \mathrm{~cm}^{3}$ (mean $5.5 \mathrm{~cm}^{3}$ ).

TR-R and IO-R revealed almost perfect agreement for TCV (TR-R ICC $=0.99,95 \%$ CI $=0.98-0.99 ; \quad$ IO-R $\mathrm{ICC}=0.98,95 \% \mathrm{CI}=0.74-0.99$ ) (Fig. 3a-c) as well as WM (TR-R ICC $=0.99,95 \% \mathrm{CI}=0.96-0.99) ; \quad$ IO-R $\mathrm{ICC}=0.97,95 \% \mathrm{CI}=0.93-0.99)$ and $\mathrm{GM}$ volumes (TR-R ICC $=0.99,95 \% \mathrm{CI}=0.97-0.99 ;$ IO-R ICC $=$ $0.96,95 \% \mathrm{CI}=0.85-0.98)$. The comparison with the fully automated segmentation yielded a good intraclass correlation ( $\mathrm{ICC}=0.86,95 \% \mathrm{CI}=0.58-0.95$ ) between ECCET and FreeSurfer results (Fig. 3d). However, visual comparison of the results of the two methods revealed that ECCET performed better, as several areas with non-brain tissue such as the venous sinuses were commonly segmented into the cerebellum using FreeSurfer without manual readjustments (see Fig. 4).

Matched-pair analysis (MS patients versus healthy volunteers)

The comparison of $\mathrm{HC}$ and MS patients showed significantly lower normalized brain volumes and normalized TCV $(p<0.05)$ as well as by trend a lower normalized cerebellar GM volume $(p=0.051)$. Details are shown in Table 1. The mean total T2 lesion volume in MS patients was $9.35 \mathrm{~cm}^{3}$ (range $0.09-30.5 \mathrm{~cm}^{3}$ ). No correlation was found between normalized TCV and clinical measures, 
Table 1 Demographic and MRI data of the case control and reliability cohort

\begin{tabular}{|c|c|c|c|}
\hline & \multicolumn{2}{|c|}{ Matched-pair analysis cohort } & \multirow{2}{*}{$\begin{array}{l}\text { Reliability cohort } \\
\text { Patients }(n=15)\end{array}$} \\
\hline & $\mathrm{HC}(n=15)$ & Patients $(n=15)$ & \\
\hline Age (years) & $35(22-62)$ & $35(21-62)$ & $49(31-67)$ \\
\hline Disease duration (years) & & $8(1-17)$ & $13(5-23)$ \\
\hline \multicolumn{4}{|l|}{ Disease course } \\
\hline RRMS $(n)$ & & 15 & 12 \\
\hline SPMS $(n)$ & & 0 & 2 \\
\hline PPMS $(n)$ & & 0 & 1 \\
\hline EDSS & & $2.7(1-4.5)$ & $2.9(0-6)$ \\
\hline Cerebellar FSS & & $0.9(0-3)$ & $1.4(0-3)$ \\
\hline $\mathrm{T} 2$ lesion volume $\left(\mathrm{cm}^{3}\right)$ & & $9.35(0.09-30.5)$ & $5.5(0.4-12.3)$ \\
\hline \multicolumn{4}{|l|}{ Raw data } \\
\hline Whole-brain volume $\left(\mathrm{cm}^{3}\right)$ & $1,095 \pm 114$ & $1,068 \pm 133$ & $1,133 \pm 133$ \\
\hline Total cerebellar volume $\left(\mathrm{cm}^{3}\right)$ & $155 \pm 14$ & $148 \pm 15$ & $143 \pm 14$ \\
\hline Cerebellar GM volume $\left(\mathrm{cm}^{3}\right)$ & $131 \pm 11$ & $126 \pm 12$ & $121 \pm 11$ \\
\hline Cerebellar WM volume $\left(\mathrm{cm}^{3}\right)$ & $24 \pm 4$ & $22 \pm 4$ & $22 \pm 5$ \\
\hline \multicolumn{4}{|l|}{ Normalized data } \\
\hline Whole-brain volume $\left(\mathrm{cm}^{3}\right)$ & $1,676 \pm 76$ & $1,606 \pm 90$ & $1,536 \pm 105$ \\
\hline Total cerebellar volume $\left(\mathrm{cm}^{3}\right)$ & $238 \pm 19$ & $224 \pm 22$ & $195 \pm 19$ \\
\hline Cerebellar GM volume $\left(\mathrm{cm}^{3}\right)$ & $202 \pm 16$ & $191 \pm 19$ & $165 \pm 16$ \\
\hline Cerebellar WM volume $\left(\mathrm{cm}^{3}\right)$ & $36 \pm 6$ & $33 \pm 6$ & $31 \pm 6.1$ \\
\hline
\end{tabular}

Clinical data and lesion volume is declared as mean value and range. Cerebellar and whole-brain volumes are declared as mean \pm standard deviation

$H C$ healthy controls, $R R M S$ relapsing remitting MS, SPMS secondary progressive MS, EDSS Expanded disability status scale, FSS functional system score (EDSS subscore)

such as $\operatorname{EDSS}(p=0.89)$, cerebellar FSS $(p=0.83)$, or disease duration $(p=0.78)$.

\section{Discussion}

Our data show that the semi-automatic method of ECCET is suitable for cerebellar segmentation in healthy controls and MS patients. The reliability study showed excellent test-retest and inter-observer reliability as well as a good intraclass correlation with FreeSurfer. Moreover, the measurements can be performed in a reasonable amount of time (on average $30 \mathrm{~min}$ per patient) with relatively low demands regarding the necessary computer infrastructure. Although of course the fully automated FreeSurfer procedure (without the use of time consuming manual adjustments) would be faster, the visual analysis of the segmentation quality of the two methods in this cohort showed that the semi-automatic segmentation with ECCET is more accurate.

Comparisons of the absolute cerebellar volumes obtained in our study to the results from previous studies [23-26] are limited because of the differences in the methodology applied, as variations in the used anatomical boundaries when separating the cerebellum from the brainstem may lead to larger or smaller cerebellar volumes. Furthermore, whether raw or normalized data are presented affects the volumes. Considering these limitations when comparing results across studies, the measured cerebellar volumes of $\mathrm{HC}$ and MS patients in our cohorts are well in the range of previous studies reporting $106-130 \mathrm{~cm}^{3}$ in MS patients and $120-150 \mathrm{~cm}^{3}$ in $\mathrm{HC}[24,25]$. It is usually considered preferable to use normalized data in cross-sectional studies of cerebral or cerebellar volumes. Our data were normalized with a normalization factor generated by the results of SIENAX when analyzing and normalizing the whole-brain volume on the basis of the Montreal Neurological Institute template (MNI; http://www.bic.mni. mcgill.ca/). This results in higher values for normalized cerebellar volume.

Although we included only relapsing-remitting MS patients in this part of our study, normalized TCV was significantly reduced in MS patients. Previous studies described significant differences in secondary and primary progressive disease types and only marginal differences in patients with clinically isolated syndrome or relapsing remitting MS $[24,25]$. Due to relatively small numbers of patients, final conclusions whether the cerebellar volume is 

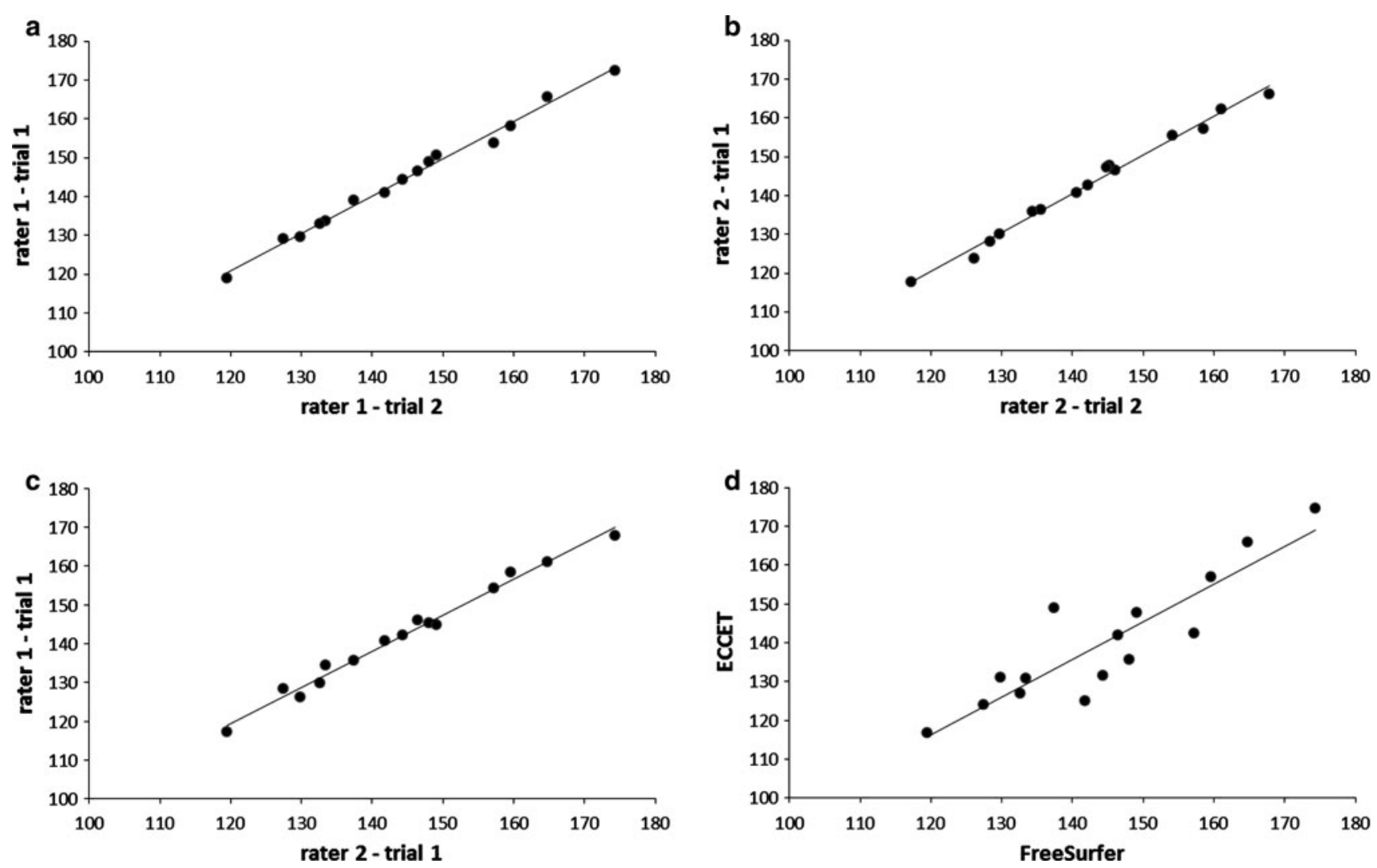

Fig. 3 Scatter plots for total cerebellar volumes showing the test-retest (a rater 1; b rater 2) and interobserver reliability $\mathbf{c}$ as well as the relationship between ECCET's semiautomated and FreeSurfer's automated measures (d). Volumes are presented as $\mathrm{cm}^{3}$

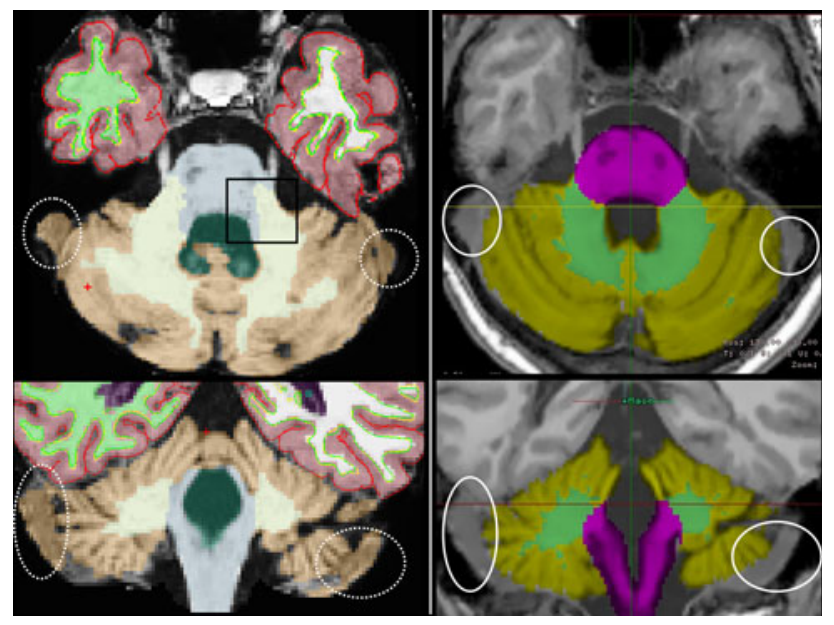

Fig. 4 Comparison of the cerebellar segmentation performed with FreeSurfer (left) versus ECCET (right). Incorrect segmentation of venous sinuses (dashed circles on the left) can be a problem when using the automated FreeSurfer software without manual corrections. No clear and reproducible cut-off at the level of the cerebellar peduncles is achieved with this software (black box)

differentially affected in patients with relapsing versus progressive forms of MS are not possible. Concerning the separate analysis of GM and WM changes, our matchedpair analysis study was probably not adequately powered, as we only found non-significant differences in cerebellar GM volume between MS patients and HC.

Whereas studies on neurodegenerative disorders showed good correlation between cerebellar volumes and degree of cerebellar ataxia [27], our study showed no correlation between cerebellar volumes and clinical data, such as the EDSS and disease duration. Here again the low sample size may be the reason for not detecting a significant correlation of cerebellar volumes and clinical scores. Moreover, in a disease that affects many different parts of the CNS in varying degrees, correlations with single clinical functions will always be confounded by other deficits.

The data in our study were acquired on 1.5-T and 3-T scanners (both Siemens) and the ECCET analysis was feasible with data from either scanner without problems. We have not systematically studied the feasibility of ECCET for segmenting cerebellar volumes using conventional 2D 3-mm T1 data. Potentially, this may be possible when interpolating the images to isotropic voxel sizes.

\section{Conclusions}

ECCET was shown to be a useful tool for cerebellar segmentation in healthy subjects and MS patients showing 
excellent test-retest and inter-observer reliability. The intraclass correlation with FreeSurfer was good while the segmented volumes were more plausible with ECCET at visual inspection. The high reliability of this method makes it a good candidate for both cross-sectional and longitudinal studies in MS. In the light of its high test-retest reliability, this method promises to be a useful tool in longitudinal studies of the relationship between cerebellar MRI and clinical data.

Conflicts of interest A. Beck has developed the ECCET Toolkit, which is currently provided free of charge for research purposes by his company 'Beck Datentechnik'. T. Derfuss served on advisory boards for Novartis, Merck-Serono, Bayer Schering, Biogen Idec, and TEVA. He received travel support from Biogen Idec, Bayer Schering, and Merck Serono. He received research and/or unrestricted grants from Novartis, Biogen Idec, Merck Serono, the German Research Foundation, the Swiss MS Society, and the European Union. L. Kappos has participated in the last 24 months as principal investigator, member or chair of planning and steering committees or advisory boards in corporate-sponsored clinical trials in multiple sclerosis and other neurological diseases. The sponsoring pharmaceutical companies for these trials include: Abbott, Actelion, Advancell, Allozyne, BaroFold, Bayer HealthCare Pharmaceuticals, Bayer Schering, Bayhill, Biogen Idec, BioMarin, CSL Behring, Elan, Genmab, Glenmark, GeNeuro SA, GlaxoSmithKline, Lilly, Merck Serono, Novartis, Novonordisk, Peptimmune, Sanofiaventis, Santhera, Roche, TEVA, UCB and Wyeth. D. Timmann received research support from the German Research Foundation (DFG), grants DFG Ti 239/9-1 and DFG TI 239/10-1, the European Union (Partner in one of the Marie Curie Initial Training Networks), the Bernd Fink Foundation and the German Heredoataxia Foundation. T. Sprenger served on advisory boards for Mitsubishi Pharma, Eli Lilly, Biogen and Allergan. He received travel support from Pfizer, Bayer Schering, Eli Lilly and Allergan. All remaining authors report no conflicts of interest.

\section{References}

1. Timmann D, Drepper J, Frings M, Maschke M, Richter S, Gerwig M, Kolb FP (2010) The human cerebellum contributes to motor, emotional and cognitive associative learning. A review. Cortex 46(7):845-857. doi:10.1016/j.cortex.2009.06.009

2. Manto M-U (ed) (2010) Cerebellar disorders : a practical approach to diagnosis and management. Cambridge University Press, Cambridge

3. Eriksson M, Andersen O, Runmarker B (2003) Long-term follow up of patients with clinically isolated syndromes, relapsingremitting and secondary progressive multiple sclerosis. Mult Scler 9(3):260-274

4. Miller DH, Hornabrook RW, Purdie G (1992) The natural history of multiple sclerosis: a regional study with some longitudinal data. J Neurol Neurosurg Psychiatr 55(5):341-346

5. Lycklama a Nijeholt G, Barkhof F (2003) Differences between subgroups of MS: MRI findings and correlation with histopathology. J Neurol Sci 206 (2):173-174

6. Ceccarelli A, Rocca MA, Pagani E, Colombo B, Martinelli V, Comi G, Filippi M (2008) A voxel-based morphometry study of grey matter loss in MS patients with different clinical phenotypes. Neuroimage 42(1):315-322

7. Mesaros S, Rovaris M, Pagani E, Pulizzi A, Caputo D, Ghezzi A, Bertolotto A, Capra R, Falautano M, Martinelli V, Comi G,
Filippi M (2008) A magnetic resonance imaging voxel-based morphometry study of regional gray matter atrophy in patients with benign multiple sclerosis. Arch Neurol 65(9):1223-1230

8. Fischl B, Salat DH, Busa E, Albert M, Dieterich M, Haselgrove C, van der Kouwe A, Killiany R, Kennedy D, Klaveness S, Montillo A, Makris N, Rosen B, Dale AM (2002) Whole brain segmentation: automated labeling of neuroanatomical structures in the human brain. Neuron 33(3):341-355

9. Tae WS, Kim SS, Lee KU, Nam EC, Kim KW (2008) Validation of hippocampal volumes measured using a manual method and two automated methods (FreeSurfer and IBASPM) in chronic major depressive disorder. Neuroradiology 50(7):569-581. doi: 10.1007/s00234-008-0383-9

10. Sanchez-Benavides G, Gomez-Anson B, Sainz A, Vives Y, Delfino M, Pena-Casanova J (2010) Manual validation of FreeSurfer's automated hippocampal segmentation in normal aging, mild cognitive impairment, and Alzheimer disease subjects. Psychiatry Res 181(3):219-225. doi:10.1016/j.pscychresns.2009.10.011

11. Richter S, Matthies K, Ohde T, Dimitrova A, Gizewski E, Beck A, Aurich V, Timmann D (2004) Stimulus-response versus stimulus-stimulus-response learning in cerebellar patients. Exp Brain Res 158(4):438-449

12. Brandauer B, Hermsdorfer J, Beck A, Aurich V, Gizewski ER, Marquardt C, Timmann D (2008) Impairments of prehension kinematics and grasping forces in patients with cerebellar degeneration and the relationship to cerebellar atrophy. Clin Neurophysiol 119(11):2528-2537. doi:10.1016/j.clinph.2008.07.280

13. Kurtzke JF (1983) Rating neurologic impairment in multiple sclerosis: an expanded disability status scale (EDSS). Neurology 33(11):1444-1452

14. Goebel R, Esposito F, Formisano E (2006) Analysis of functional image analysis contest (FIAC) data with brainvoyager QX: from single-subject to cortically aligned group general linear model analysis and self-organizing group independent component analysis. Hum Brain Mapp 27(5):392-401. doi:10.1002/hbm.20249

15. Aurich VaW J (1995) Non-linear Gaussian filters performing edge preserving diffusion. In: Proceedings 17. DAGM-Symposium, Springer, Bielefeld, pp 538-545

16. Aurich V, Winkler G, Liebscher V (1999) Probabilistic image smoothing: recent advances. In: Benes V, Janacek J, Saxl I (eds) Proceedings S4G, International Conference on Stereology, Spatial Statistics and Stochastic Geometry, Prague. Union of Czech Mathematicians and Physicists, pp 273-278

17. Beck A (2003) Ein System zur Verarbeitung und Visualisierung von Voxeldaten. Heinrich-Heine University of Düsseldorf, Düsseldorf

18. Aurich V, Beck A (2002) ECCET: Ein System zur 3D-Visualisierung von Volumendaten mit Echtzeitnavigation. In: Proceedings Workshops über Bildverarbeitung für die Medizin, Leipzig, pp 389-392

19. Bendfeldt K, Kuster P, Traud S, Egger H, Winklhofer S, MuellerLenke N, Naegelin Y, Gass A, Kappos L, Matthews PM, Nichols TE, Radue EW, Borgwardt SJ (2009) Association of regional gray matter volume loss and progression of white matter lesions in multiple sclerosis - a longitudinal voxel-based morphometry study. Neuroimage 45(1):60-67. doi:10.1016/j.neuroimage.2008.10.006

20. Jasperse B, Valsasina P, Neacsu V, Knol DL, De Stefano N, Enzinger C, Smith SM, Ropele S, Korteweg T, Giorgio A, Anderson V, Polman CH, Filippi M, Miller DH, Rovaris M, Barkhof F, Vrenken $H$ (2007) Intercenter agreement of brain atrophy measurement in multiple sclerosis patients using manually-edited SIENA and SIENAX. J Magn Reson Imag 26(4):881-885

21. Shrout PE, Fleiss JL (1979) Intraclass correlations-uses in assessing rater reliability. Psychol Bull 86(2):420-428

22. McGraw KO, Wong SP (1996) Forming inferences about some intraclass correlation coefficients. Psychol Methods 1(1):30-46 
23. Dimitrova A, Gerwig M, Brol B, Gizewski ER, Forsting M, Beck A, Aurich V, Kolb FP, Timmann D (2008) Correlation of cerebellar volume with eyeblink conditioning in healthy subjects and in patients with cerebellar cortical degeneration. Brain Res 1198:73-84. doi:10.1016/j.brainres.2008.01.034

24. Calabrese M, Mattisi I, Rinaldi F, Favaretto A, Atzori M, Bernardi V, Barachino L, Romualdi C, Rinaldi L, Perini P, Gallo P (2011) Magnetic resonance evidence of cerebellar cortical pathology in multiple sclerosis. J Neurol Neurosurg Psychiatr 81(4):401-404

25. Anderson VM, Fisniku LK, Altmann DR, Thompson AJ, Miller DH (2009) MRI measures show significant cerebellar gray matter volume loss in multiple sclerosis and are associated with cerebellar dysfunction. Mult Scler 15(7):811-817

26. Dimitrova A, Zeljko D, Schwarze F, Maschke M, Gerwig M, Frings M, Beck A, Aurich V, Forsting M, Timmann D (2006) Probabilistic 3D MRI atlas of the human cerebellar dentate/interposed nuclei. Neuroimage 30(1):12-25. doi:10.1016/j.neuroimage.2005.09.020

27. Richter S, Dimitrova A, Maschke M, Gizewski E, Beck A, Aurich V, Timmann D (2005) Degree of cerebellar ataxia correlates with three-dimensional MRI-based cerebellar volume in pure cerebellar degeneration. Eur Neurol 54(1):23-27. doi:10.1159/000087241 